\title{
Young People's Smoking Behaviour; Determinants and Health Consequences: Evidence from Cape Area Panel Study (CAPS)
}

\author{
Amos Olugbenga Oyedokun \\ Demography and Social Statistics, Faculty of Social Sciences, Obafemi Awolowo University, Ile-Ife, Osun State, Nigeria \\ Email: aooyedokun \{at\} yahoo.com
}

\begin{abstract}
This paper examined the determinants and health-consequences of smoking among youths in Cape Town, South Africa between 2002 and 2005. Findings show that 25\% of the respondents had started smoking cigarette while 3\% had started smoking dagga or other drug at the baseline. More than a third of those who smoked cigarette and about a third of those who smoked dagga or other drugs in 2002 were less than 18 years. Being a male and a coloured, and living in predominantly coloured neighbourhoods significantly associated with smoking cigarette and dagga/other drugs in past month. Childhood place of residence being urban and living in poor neighbourhoods significantly associates with smoking of cigarette and dagga. Also, those who have initiated cigarette smoking at the first wave were significantly more likely to self-report smoking-related illnesses in the three waves. Therefore, program and policy interventions should be designed to address such behaviour.
\end{abstract}

Keywords--- Smoking, Determinants, Health Consequences, Young People in Cape Town

\section{INTRODUCTION}

Use of tobacco has been tagged one of the most modifiable risk factors and preventable cause of death in the world. The World Health Organization (WHO) has attributed some four million deaths a year to tobacco with the possibility of an increase into the future. More than a decade ago, it was estimated that by 2030, smoking will kill one in six people globally if current trends continue $[1,2]$ and this will include seven million people in the developing countries [3]. Smoking is a major drain on poor people's incomes as poor people were found to spend a larger proportion of their income on cigarette [4] and a huge cause of ill health and premature death [5]. Young people's smoking behaviour has been identified as a public health issue because of the immediate and long-term health consequences such as increased risk of cardiovascular diseases, respiratory illnesses, tuberculosis, cancer and other smoking-related illnesses $[6,7,8,9$, $10]$.

Negative health consequences accruing from tobacco use result not only from direct consumption of tobacco but also from exposure to second-hand smoke [11] with almost six million people dying from tobacco use and exposure annually- more males than females $[12,13]$. The rising levels of tobacco use among young people in the 1990s led to smoking being labelled a paediatric disease or epidemic [14]. Around 1999, adolescent smokers were above the global average at $23 \%$ compared to $20 \%$ but reportedly dropped to $18.5 \%$ in $2002[15,16]$, a rate still very high needing serious attention. Smoking has been identified as a serious problem in Western Cape where prevalence was proportionally higher than that of South Africa as a whole [6, 17]. Cardiovascular disease closely related to tobacco use has been found to be the second largest cause of death in South Africa (16.6\% in 2000) [18]. This has not changed significantly since it was first reported [19]. There are several studies in the area of adolescent smoking behaviour in South Africa ([20, 21, 14, 22, $23,24,16,4,25]$, while some investigated the prevalence and determinants of smoking behaviour [4, 20, 23, 26], others examined the issue in relation to smoking cessation or effect $[20-21,16]$.

With respect to the prevalence of cigarette smoking and factors influencing the behaviour, a study in South Africa found that a little less than two-fifth of adolescents had tried smoking in their lifetime-37.6\% and that a little less than one-fifth were past month or current smokers-18.5\% [15]. A study reported that up to $29.5 \%$ of adolescents had smoked, with $21 \%$ admitting to being current smokers (more than 1 per day in the last month preceding the survey) which was almost double that of global prevalence estimates [26, 27]. In a recent report by Healthy Active Kids South Africa (2010), it was noted that smoking among South African adolescents has remained constant at a high level since 2007 taking into consideration the fantastic tobacco legislation in South Africa. According to the report, prevalence is generally higher in males than females, and higher in urban areas, especially the Western Cape. Also, the majority of smokers start smoking before the age of 19 years with $6.8 \%$ starting under the age of 10 years [19]. Madu and Matla (2004) earlier found a prevalence rate of $10.6 \%$ among the adolescents studied and that males are more likely to engage in the behaviour when compared to their female counterparts. According to the findings from the same study, boredom, 
tiredness, stress and parties were the reasons for indulging in smoking behaviour while the mean age for the first smokers was fifteen years [24].

Other individual factors that have been found to influence adolescent smoking behaviour include personality, attitudes and a sense of well-being [28, 29]. It had been noted that delaying the age when kids first experiment or begin using tobacco can reduce the risk that they become regular or daily tobacco users and increase their chances of successfully quitting, if they do become regular users [30]. Also, some researchers have found in a different context that the prevalence of smoking increased with age and that smoking associate strongly with alcohol drinking and use of cannabis among adolescents [31]. Another study showing racial differences in inclination to smoke revealed that young Africans were the least inclined, followed by Indians and then Coloureds while Whites were the most inclined to be smokers [25]. According to Panday et al. (2003) [20], poverty was linked to high level of smoking, although van Walbeek (2002) found that there was a decrease in smoking prevalence between 1993 and 2000 for most demographic and socio-economic groups due to sharp increase in cigarette prices; young adults, the Coloureds, Whites, females and high income households still maintained relatively high smoking prevalence [4]. In a similar vein, Guindon (2009) found that wealth (measured by asset index) is statistically significantly associated with the onset of smoking and that individuals in higher wealth categories tend to initiate smoking earlier compared to those in the bottom quintile [32].

Some of the socio-cultural factors linked to smoking behaviour among young South Africans include peer substance abuse, parental affection or lack of it, ethnic identification, discrimination and victimisation. Panday et al. (2003) reported that while "Coloured" and "Black" participants were influenced by smoking peers, the "White" participants self-reported that they smoked by choice [20]. Brook et al. (2006) found that parental affection reduced the odds of being a regular smoker compared with experimental or non-smokers; peer substance abuse predicted an increase in the risk of being a regular smoker compared to their counterparts; ethnic identification predicted a decrease in the risk of being a regular smoker compared to their counterparts; while discrimination and victimisation predicted an increase in the risk of being an experimental smoker compared with a non-smoker among the adolescents studied in Johannesburg [23]. In a similar vein, Guindon (2009) in a different context found that youths who have friends that smoke have about 15 times higher hazard of smoking when compared to their non-smoking counterparts. Also, belonging to mass organisations or clubs and having ever worked to earn money help to delay smoking onset (14.7\% and $25.7 \%$ respectively), while literacy and access to money increased the odds of initiating smoking [32].

Some studies have found that although there is peer influence on adolescents to smoke, there are evidences that the influence of family is stronger (prevalence of adult smoking) [28, 33]. In line with the above finding, more current adolescent smokers (42.5\%) as compared to non-smokers (23.2\%) had one (or more) parent and/or guardian who smoke [26], and a conflicting relationship with parents has been shown to influence smoking behaviour in adolescents [29]. King et al. (2003) found that having a personal knowledge of adults who engaged in antisocial behaviour associate strongly with the adolescents smoking behaviour [34]. It has however been reported that awareness of some of the dangers posed by smoking is low among Black and White South Africans [22]. Therefore it is necessary to seek answers to the following crucial questions: (a) Are adolescents and young adults engaging in smoking behaviour in the study area? (b) What are the predictors of smoking behaviours among these adolescents and young people using a longitudinal design? (c) Is there any health consequence arising from their smoking behaviour in the study area? Although there have been several studies on adolescent smoking behaviour in South Africa, a close scrutiny of these past studies showed that they are largely cross-sectional with its attendant limitations. Also, studies linking smoking behaviour to its health consequences are scant in the study area, hence, the main motivation for this study.

\section{DATA AND METHODS}

This paper makes use of the first three waves of secondary data collected from the Cape Area Panel Study (CAPS) between 2002 and 2005. The Cape Area Panel Study (CAPS) is a longitudinal study of the lives of about 4,800 adolescents and young adults randomly selected in metropolitan Cape Town, South Africa. The first wave of the study collected complete information from 4,752 randomly selected young people aged 14-22 years between August and December, 2002. Wave 1 also collected information on all members of these young people's households, as well as a random sample of households that did not have members aged 14-22. A third of the youth sample was re-interviewed again around July 2003 (Wave 2a) while the remaining two-thirds were re-visited and interviewed before December 2004 (Wave 2b). The goal of Wave 2 was, first, to keep in contact with CAPS young adult respondents who had completed Wave 1 questionnaires during the three-year period before Wave 3, which was planned for 2005, and then to update areas of core panel data, such as employment, schooling and household rosters, as well as to introduce new modules to probe selected topics in further detail (See Lam et al. 2008 for details on CAPS dataset) [35].

The reported results in this paper are based on the analysis of unweighted sample of youths interviewed between 2002 and 2005. The data if weighted are representative of the entire cohort of adolescents and young adults in Cape Town because it has been found in earlier studies that weighting for attrition between 2002 and 2005 does not change any 
results substantially [36]. This is a confirmation of earlier findings using data from different settings including South Africa that attrition is never a general problem for obtaining consistent estimates even though it has been intuitively believed by many analysts that attrition is likely to be selective on characteristics such as schooling, and thus that high attrition is likely to bias estimates [37]. The reasons for attrition among CAPS respondents include movement to other areas within South Africa or even abroad, sicknesses, crime leading to imprisonment, and death.

\subsection{Variables}

The independent variables used for this study include age, sex, race/population group, educational level and work status. Other contextual independent variables include religious affiliation, childhood place of residence, neighbourhood racial concentration and family structure; school attendance, school environment, participation in prosocial activities, neighbourhood socio-economic status (SES), childhood neighbourhood type and alcohol consumption in past month to the time of the survey. The proxy questions used for the outcome variable(s) are: Do you smoke cigarette in past month (to the time of the survey)? And do you smoke Dagga/other drugs in past month (to the time of the survey)? What are your health problems? Responses to the question on health problems were then recategorised based on evidence from literatures into smoking-related illnesses (if the respondents reported tuberculosis or other respiratory problems) and otherwise (if other sicknesses which are not related were reported by the respondents)

\subsection{Analyses}

The study employed the univariate method of analysis to generate the profile of the respondents (frequency tables), bivariate analysis (cross-tabulations using chi-square tests of associations as a measure of goodness of fit), and binary logistic regression model. The data for this study was analysed using Stata/SE 12 analytical package [38]. The binary logistic regression model is of the following form:

$$
\operatorname{In}(\mathrm{ODDS})=\operatorname{In}[\hat{\mathrm{Y}} / 1-\hat{\mathrm{Y}}]=\mathrm{a}+\mathrm{b}_{1} \mathrm{X}_{1}+\mathrm{b}_{2} \mathrm{X}_{2}+\ldots+\mathrm{b}_{\mathrm{n}} \mathrm{X}_{\mathrm{n}}+\mathrm{E}
$$

where $\hat{Y}$ is the predicted probability of the event which is coded 1 (smoked in past month) rather than with 0 (never smoked in past month), $1-\hat{Y}$ is the predicted probability of never smoked in the past month, and $\mathrm{X}_{\mathrm{s}}$ are the predictors fitted in the model. This model is also applicable to those reporting smoking-related illnesses coded as 1 and those who did not report any smoking-related illnesses coded as 0 . The responsible ethical review bodies at the University of Cape Town and University of Michigan approved the CAPS projects designs and Wave 1-4 instruments [39] and the data used for the analyses in this paper is in the public domain.

\section{RESULTS}

Table 1: Distribution of the respondents by background characteristics (CAPS 2002-2005)

\begin{tabular}{|l|l|l|l|}
\hline Variable(s) & Wave 1 (2002) & Wave 2 (2003/04) & Wave 3 (2005) \\
\hline Independent variables & \multicolumn{3}{|l|}{} \\
\hline Age & \multicolumn{3}{|l|}{} \\
\hline Less than 18 years & $51.68(1,659)$ & $34.99(381)$ & $13.30(402)$ \\
\hline 18 years and above & $48.32(1,551)$ & $65.01(708)$ & $86.70(2,621)$ \\
\hline Total & $100.00(3,210)$ & $100.00(1,089)$ & $100.00(3,023)$ \\
\hline Mean age of the respondents & 17 years & 19 years & 20 years \\
\hline Sex & \multicolumn{3}{|l|}{} \\
\hline Male & $46.14(1,481)$ & $45.59(978)$ & $46.17(1,482)$ \\
\hline Female & $53.86(1,729)$ & $54.41(1,167)$ & $53.83(1,728)$ \\
\hline Total & $100.00(3,210)$ & $100.00(2,145)$ & $100.00(3,210)$ \\
\hline Race/ population group & \multicolumn{3}{|l|}{} \\
\hline \multicolumn{1}{|c|}{ Black/African } & $43.89(1,409)$ & $39.71(851)$ & $43.86(1,407)$ \\
\hline Coloured & $47.26(1,517)$ & $49.93(1,070)$ & $86.91(1,505)$ \\
\hline White & $8.85(284)$ & $9.61(206)$ & $0.41(13)$ \\
\hline Indian/Asian & - & $0.75(16)$ & $100.00(3,208)$ \\
\hline Total & $100.00(3,210)$ & $100.00(2,143)$ & - \\
\hline Educational level & \multicolumn{2}{|l|}{} \\
\hline No formal education & - & - & - \\
\hline Primary & $21.48(688)$ & $4.48(91)$ & \\
\hline
\end{tabular}




\begin{tabular}{|l|c|l|c|}
\hline Secondary & $75.30(2,412)$ & $79.08(1,607)$ & $67.45(833)$ \\
\hline Tertiary & $3.22(103)$ & $16.44(334)$ & $32.55(402)$ \\
\hline Total & $100.00(3,203)$ & $100.00(2,032)$ & $100.00(1,235)$ \\
\hline Current work status & \multicolumn{3}{|l|}{} \\
\hline Yes & $52.07(540)$ & $27.85(596)$ & $39.41(1,264)$ \\
\hline No & $47.93(497)$ & $72.15(1,544)$ & $60.59(1,943)$ \\
\hline Total & $100.00(1,037)$ & $100.00(2,140)$ & $100.00(3,207)$ \\
\hline
\end{tabular}

Figures in parentheses are frequencies.

Table 1 presents the profile of the young respondents followed from Wave 1 in 2002 through to Wave 3 in 2005 . The result shows that at the baseline, more than half of the respondents were less than 18 years of age, and about one-third at the second wave in 2003/2004, while about one-tenth were less than 18 years of age at the third wave in 2005. Generally, observing the three waves between 2002 and 2005, more females than males were sampled, while the majority of the sample were Coloured, followed by Africans. Most of the adolescents and young adults interviewed had some secondary education at the three waves of data collection, while a little less than half were not working at the baseline survey in 2002; that proportion increased to $72 \%$ at the second wave in 2003/2004 and 61\% at the third wave in 2005.

Table 2: Percentage distribution of respondents by contextual factors (CAPS 2002-2005)

\begin{tabular}{|c|c|c|c|}
\hline Variable(s) & Wave 1 (2002) & Wave $2(2003 / 04)$ & Wave 3 (2005) \\
\hline \multicolumn{4}{|l|}{ Contextual factors ${ }^{I}$} \\
\hline \multicolumn{4}{|l|}{ Religious affiliation } \\
\hline None & $11.21(357)$ & - & - \\
\hline Christianity & $56.33(1,794)$ & - & - \\
\hline Islam & $10.11(322)$ & - & - \\
\hline Others & $22.35(712)$ & - & - \\
\hline Total & $100.00(3,185)$ & - & - \\
\hline \multicolumn{4}{|c|}{ Childhood place of residence } \\
\hline Urban & $81.96(2,612)$ & - & - \\
\hline Rural & $18.04(575)$ & - & - \\
\hline Total & $100.00(3,187)$ & - & - \\
\hline \multicolumn{4}{|c|}{ Neighbourhood racial concentration } \\
\hline African & $43.83(1,407)$ & - & - \\
\hline Coloured & $45.14(1,449)$ & - & - \\
\hline White & $11.03(354)$ & - & - \\
\hline Total & $100.00(3,210)$ & - & - \\
\hline \multicolumn{4}{|l|}{ Family structure } \\
\hline No-parent family & $20.26(650)$ & - & $19.91(615)$ \\
\hline Single-parent family & $37.89(1,216)$ & - & $41.44(1,280)$ \\
\hline Both-parents family & $41.85(1,343)$ & - & $38.65(1,194)$ \\
\hline Total & $100.00(3,209)$ & - & $100.00(3,089)$ \\
\hline \multicolumn{4}{|l|}{ School attendance } \\
\hline Yes & $68.31(2,192)$ & $58.17(616)$ & $36.75(1,176)$ \\
\hline No & $31.69(1,017)$ & $41.83(443)$ & $63.25(2,024)$ \\
\hline Total & $100.00(3,209)$ & $100.00(1,059)$ & $100.00(3,200)$ \\
\hline \multicolumn{4}{|l|}{ School environment } \\
\hline Non-problematic & $24.08(773)$ & - & - \\
\hline Problematic & $75.92(2,437)$ & - & - \\
\hline Total & $100.00(3,210)$ & - & - \\
\hline \multicolumn{4}{|c|}{ Participation in prosocial activities } \\
\hline Non-participants & $42.94(1,378)$ & - & - \\
\hline Participants & $57.06(1,831)$ & - & - \\
\hline Total & $100.00(3,209)$ & - & - \\
\hline Neighbourhood SES ${ }^{2}$ & & & \\
\hline
\end{tabular}

\footnotetext{
${ }^{1}$ Contextual factors are factors serving as facilitators or impediments toward a particular behaviour.

${ }^{2}$ Measure of poverty at the Magisterial district level
} 


\begin{tabular}{|l|l|l|l|}
\hline Affluent & $30.12(967)$ & - & - \\
\hline Poor & $69.88(2,243)$ & - & - \\
\hline Total & $100.00(3,210)$ & - & - \\
\hline Childhood neighbourhood type $^{3}$ & & & \\
\hline Non-problematic & $74.07(2,377)$ & - & - \\
\hline Problematic & $25.93(832)$ & - & - \\
\hline Total & $100.00(3,209)$ & - & - \\
\hline Consumed Alcohol in past month & & & \\
\hline Yes & $20.41(653)$ & - & $37.67(1,208)$ \\
\hline No & $79.59(2,547)$ & - & $62.33(1,999)$ \\
\hline Total & $100.00(3,200)$ & - & $100.00(3,207)$ \\
\hline
\end{tabular}

Figures in parentheses are frequencies. Overall samples vary in some instances due to missing values. - indicates that the question was not asked during the wave in question.

Table 2 above presents the percentage distributions of the respondents according to the measures of contextual factors. The table reveals that at the baseline in 2002 more than half of the respondents were Christian. About one-fifth of the young respondents had no parent in 2002 while more than one-third was from single-parent families at both the first and third waves of data collection. The majority of the respondents reported a problematic school environment while the proportion of young people attending school decreased consistently between Waves 1 and 2, and between Waves 2 and 3 .

A little below half of the respondents lived in predominantly Coloured neighbourhoods (45\%) followed by $44 \%$ living in Black/African neighbourhoods in 2002, with more than half indicating that they participated in one prosocial activity or the other (57\%). Close to seventy percent lived in relatively poor neighbourhoods in 2002. In 2005, onequarter of the respondents reported that they were exposed to problematic neighbourhoods when growing up, with the majority having grown up in an urban place of residence, while there was an increase in the proportion of young people that consumed alcohol in past month from 20\% to 38\% between 2002 and 2005 respectively.

Table 3a: Distribution of the respondents by smoking behaviour (CAPS 2002-2005)

\begin{tabular}{|l|l|l|l|l|}
\hline \multirow{2}{*}{ Outcome Variables } & Wave 1 & \multicolumn{2}{l|}{ Wave 3 } \\
\cline { 2 - 5 } & Frequency & Percent & Frequency & Percent \\
\hline Smoked cigarette in past month? & & & & \\
\hline Yes & 813 & 25.42 & 1,147 & 35.77 \\
\hline No & 2,385 & 74.58 & 2,060 & 64.23 \\
\hline Total & 3,198 & 100.00 & 3,207 & 100.00 \\
\hline $\begin{array}{l}\text { Smoked dagga/other drugs in past } \\
\text { month? }\end{array}$ & & & \\
\hline Yes & 106 & 3.31 & 195 & 6.08 \\
\hline No & 3,095 & 96.69 & 3,012 & 93.92 \\
\hline Total & 3,201 & 100.00 & 3,207 & 100.00 \\
\hline
\end{tabular}

Overall samples vary in some instances due to missing values. Dagga= local name for Marijuana

Table 3 a reveals that one-quarter of the young respondents are current smokers of cigarette at the baseline, and the proportion increased to more than one-third at the third wave of data in 2005. Those who were current smokers of dagga (Marijuana) or other drugs increased from 3\% to 6\% between 2002 and 2005.

Table $3 \mathrm{~b}$ shows the distribution of the adolescents and young respondents by their responses to question on main health problems especially for those who reported that they had one health problem or the other. Those who had tuberculosis ranged between 4 to 5 per cent in the study area, and those with other respiratory problems raged between 32 per cent and 36 per cent between 2002 and 2005.

\footnotetext{
${ }^{3}$ Availability of problem drinkers, drug addict and criminals when growing up?
} 
Table 3b: Distribution of the respondents by self-reported main health problems/disability (CAPS 2002-2005)

\begin{tabular}{|l|l|l|l|}
\hline Main health & Wave 1 & Wave 2 & Wave 3 \\
\cline { 2 - 4 } problem/disability & Percent (frequency) & Percent (frequency) & Percent (frequency) \\
\hline Tuberculosis & $3.83(11)$ & $5.43(7)$ & $4.67(10)$ \\
\hline Other respiratory problems & $32.06(92)$ & $36.43(47)$ & $35.51(76)$ \\
\hline Physically handicapped & $3.48(10)$ & $1.55(2)$ & $2.80(6)$ \\
\hline $\begin{array}{l}\text { Problems with sight, } \\
\text { hearing or speech }\end{array}$ & $16.03(46)$ & $4.65(6)$ & $11.21(24)$ \\
\hline Mental problem & $1.74(5)$ & $6.20(8)$ & $6.07(13)$ \\
\hline HIV/AIDS & - & $1.55(2)$ & $3.27(7)$ \\
\hline$*$ Others (specify) & $42.86(123)$ & $44.19(57)$ & $36.45(78)$ \\
\hline Total & $100.00(287)$ & $100.00(129)$ & $100.00(214)$ \\
\hline
\end{tabular}

*This includes various illnesses lumped together and some of those terms were ambiguous

Table 4: Pearson chi-square test results showing the association between the selected predictor variables and those who smoked cigarette and dagga/other drugs in past month (CAPS 2002-2005)

\begin{tabular}{|c|c|c|c|c|}
\hline \multirow{2}{*}{$\begin{array}{l}\text { Personal/Background } \\
\text { Characteristics }\end{array}$} & \multicolumn{2}{|c|}{ Cigarette smoking } & \multicolumn{2}{|c|}{ Dagga/other drugs } \\
\hline & Wave 1 & Wave 3 & Wave 1 & Wave 3 \\
\hline \multicolumn{5}{|l|}{ Age } \\
\hline Less than 18 years & $38.87(316)$ & $10.82(119)$ & $33.96(36)$ & $14.29(27)$ \\
\hline 18 years and above & $61.13(497)$ & $89.18(981)$ & $66.04(70)$ & $85.71(162)$ \\
\hline Total & $100.00(813)$ & $100.00(1,100)$ & $100.00(106)$ & $100.00(189)$ \\
\hline Pearson coefficient & $71.0461 * * *$ & $9.3194 * * *$ & $13.7679 * * *$ & 0.1659 \\
\hline \multicolumn{5}{|l|}{ Sex } \\
\hline Male & $60.52(492)$ & $60.68(696)$ & $77.36(82)$ & $79.49(155)$ \\
\hline Female & $39.48(321)$ & $39.32(451)$ & $22.64(24)$ & $20.51(40)$ \\
\hline Total & $100.00(813)$ & $100.00(1,147)$ & $100.00(106)$ & $100.00(195)$ \\
\hline Pearson coefficient & $90.0863 * * *$ & $151.7152 * * *$ & $42.9890 * * *$ & $92.8549 * * *$ \\
\hline \multicolumn{5}{|l|}{ Race/ population group } \\
\hline Black/African & $16.97(138)$ & $21.03(241)$ & $22.64(24)$ & $25.64(50)$ \\
\hline Coloured & $75.15(611)$ & $70.16(804)$ & $70.75(75)$ & $66.67(130)$ \\
\hline White & $7.87(64)$ & $8.46(97)$ & $6.60(7)$ & $7.18(14)$ \\
\hline Indian/Asian & - & $0.35(4)$ & - & $0.51(1)$ \\
\hline Total & $100.00(813)$ & $100.00(1,146)$ & $100.00(106)$ & $100.00(195)$ \\
\hline Pearson coefficient & $360.6842 * * *$ & $418.3503 * * *$ & $24.8221 * * *$ & $33.7326 * * *$ \\
\hline \multicolumn{5}{|l|}{ Educational level } \\
\hline No formal education & - & - & - & - \\
\hline Primary & $17.63(143)$ & - & $24.53(26)$ & - \\
\hline Secondary & $78.67(638)$ & $61.90(169)$ & $73.58(78)$ & $78.26(36)$ \\
\hline Tertiary & $3.70(30)$ & $38.10(104)$ & $1.89(2)$ & $21.74(10)$ \\
\hline Total & $100.00(811)$ & $100.00(273)$ & $100.00(106)$ & $100.00(46)$ \\
\hline Pearson coefficient & $9.6857 * * *$ & $5.0103 * *$ & 1.1298 & 2.5204 \\
\hline \multicolumn{5}{|l|}{ Current work status } \\
\hline Yes & $53.72(238)$ & $49.04(562)$ & $45.00(27)$ & $36.41(71)$ \\
\hline No & $46.28(205)$ & $50.96(584)$ & $55.00(33)$ & $63.59(124)$ \\
\hline Total & $100.00(443)$ & $100.00(1,146)$ & $100.00(60)$ & $100.00(195)$ \\
\hline Pearson coefficient & 0.8421 & $69.1509 * * *$ & 1.2616 & 0.7874 \\
\hline \multicolumn{5}{|l|}{ Contextual Factors } \\
\hline \multicolumn{5}{|l|}{ Religious affiliation } \\
\hline None & $10.40(84)$ & $10.65(121)$ & $14.42(15)$ & $14.06(27)$ \\
\hline Christianity & $57.30(463)$ & $58.45(664)$ & $54.81(57)$ & $57.29(110)$ \\
\hline Islam & $17.82(144)$ & $14.26(162)$ & $13.46(14)$ & $11.98(23)$ \\
\hline Others & $14.48(117)$ & $16.64(189)$ & $17.31(18)$ & $16.67(32)$ \\
\hline Total & $100.00(808)$ & $100.00(1,136)$ & $100.00(104)$ & $100.00(192)$ \\
\hline Pearson coefficient & $94.5281 * * *$ & $57.8248 * * *$ & 3.5022 & 5.2184 \\
\hline
\end{tabular}




\begin{tabular}{|c|c|c|c|c|}
\hline \multicolumn{5}{|c|}{ Childhood place of residence } \\
\hline Urban & $91.30(735)$ & $91.20(1,036)$ & $92.38(97)$ & $93.81(182)$ \\
\hline Rural & $8.70(70)$ & $8.08(100)$ & $7.62(8)$ & $6.19(12)$ \\
\hline Total & $100.00(805)$ & $100.00(1,136)$ & $100.00(105)$ & $100.00(194)$ \\
\hline Pearson coefficient & $64.1089 * * *$ & $102.2572 * * *$ & $7.9639 * * *$ & $19.6818 * * *$ \\
\hline \multicolumn{5}{|c|}{$\begin{array}{lc}\begin{array}{l}\text { Neighbourhood } \\
\text { concentration }\end{array} & \text { racial } \\
\end{array}$} \\
\hline African & $18.08(147)$ & $21.71(249)$ & $21.70(23)$ & $26.15(51)$ \\
\hline Coloured & $71.09(578)$ & $66.70(765)$ & $67.92(72)$ & $64.62(126)$ \\
\hline White & $10.82(88)$ & $11.60(133)$ & $10.38(11)$ & $9.23(18)$ \\
\hline Total & $100.00(813)$ & $100.00(1,147)$ & $100.00(106)$ & $100.00(195)$ \\
\hline Pearson coefficient & $327.7451 * * *$ & $383.1680 * * *$ & $25.0156^{* * *}$ & $32.8104 * * *$ \\
\hline \multicolumn{5}{|l|}{ Family structure } \\
\hline No-parent family & $18.45(150)$ & $18.14(203)$ & $17.92(19)$ & $16.58(31)$ \\
\hline Single-parent family & $38.25(311)$ & $42.45(475)$ & $43.40(46)$ & $47.06(88)$ \\
\hline Both-parents family & $43.30(352)$ & $39.41(441)$ & $38.68(41)$ & $36.36(68)$ \\
\hline Total & $100.00(813)$ & $100.00(1,119)$ & $100.00(106)$ & $100.00(187)$ \\
\hline Pearson coefficient & 2.3573 & 3.4762 & 1.4373 & 2.9156 \\
\hline \multicolumn{5}{|l|}{ School attendance } \\
\hline Yes & $42.68(347)$ & $22.08(253)$ & $33.96(36)$ & $20.00(39)$ \\
\hline No & $57.32(466)$ & $77.92(893)$ & $66.04(70)$ & $80.00(156)$ \\
\hline Total & $100.00(813)$ & $100.00(1,146)$ & $100.00(106)$ & $100.00(195)$ \\
\hline Pearson coefficient & $327.9919 * * *$ & $165.5267 * * *$ & $59.6193 * * *$ & $25.0743 * * *$ \\
\hline \multicolumn{5}{|l|}{ School environment } \\
\hline Non-problematic & $23.62(192)$ & $23.02(264)$ & $25.47(27)$ & $17.95(35)$ \\
\hline Problematic & $76.38(621)$ & $76.98(883)$ & $74.53(79)$ & $82.05(160)$ \\
\hline Total & $100.00(813)$ & $100.00(1,147)$ & $100.00(106)$ & $100.00(195)$ \\
\hline Pearson coefficient & 0.1269 & 1.0265 & 0.1151 & $4.2201 * *$ \\
\hline \multicolumn{5}{|c|}{ Participation in prosocial activities } \\
\hline Non-participants & $56.09(456)$ & $51.44(590)$ & $70.75(75)$ & $54.87(107)$ \\
\hline Participants & $43.91(357)$ & $48.56(557)$ & $29.25(31)$ & $45.13(88)$ \\
\hline Total & $100.00(813)$ & $100.00(1,147)$ & $100.00(106)$ & $100.00(195)$ \\
\hline Pearson coefficient & $76.6253 * * *$ & $52.9062 * * *$ & $34.6596 * * *$ & $12.1073 * * *$ \\
\hline \multicolumn{5}{|c|}{ Neighbourhood SES ${ }^{4}$} \\
\hline Affluent & $40.96(333)$ & $38.54(442)$ & $37.74(40)$ & $39.49(77)$ \\
\hline Poor & $59.04(480)$ & $61.46(705)$ & $62.26(66)$ & $60.51(118)$ \\
\hline Total & $100.00(813)$ & $100.00(1,147)$ & $100.00(106)$ & $100.00(195)$ \\
\hline Pearson coefficient & $60.9453 * * *$ & $60.0549 * * *$ & 2.9983 & $8.6522 * * *$ \\
\hline \multicolumn{5}{|c|}{ Childhood neighbourhood type ${ }^{5}$} \\
\hline Non-problematic & $64.58(525)$ & $68.35(784)$ & $51.89(55)$ & $60.51(118)$ \\
\hline Problematic & $35.42(288)$ & $31.65(363)$ & $48.11(51)$ & $39.49(77)$ \\
\hline Total & $100.00(813)$ & $100.00(1,147)$ & $100.00(106)$ & $100.00(195)$ \\
\hline Pearson coefficient & $50.8787 * * *$ & $30.1578 * * *$ & $27.9899 * * *$ & $19.7961 * * *$ \\
\hline \multicolumn{5}{|c|}{ Consumed Alcohol in past month } \\
\hline Yes & $46.73(379)$ & $37.05(425)$ & $61.32(65)$ & $28.72(56)$ \\
\hline No & $53.27(432)$ & $62.95(722)$ & $38.68(41)$ & $71.28(139)$ \\
\hline Total & $100.00(811)$ & 100.00() $1,147$ & $100.00(106)$ & $100.00(195)$ \\
\hline Pearson coefficient & $463.6843 * * *$ & $486.0048^{* * * *}$ & $112.5099 * * *$ & $99.9192 * * *$ \\
\hline
\end{tabular}

Significant at $* * * \mathrm{p}<0.01 ; * * \mathrm{p}<0.05$

From Table 4, it is clear that more than one-third of the respondents who were less than 18 years smoked cigarette at the baseline (2002) and it reduced to about one-tenth at the third wave (2005) with more males smoking cigarette at both waves $(61 \%)$. Current cigarette smokers at both waves were of Coloured race with majority having some secondary education; with more than half being Christians. Majority of the current smokers grew up in urban centres with high concentration of people of Coloured descent and they were out of school with more than half of them not

\footnotetext{
${ }^{4}$ Measure of poverty at the Magisterial district level

${ }^{5}$ Availability of problem drinkers, drug addict and criminals when growing up?
} 
participating in prosocial activities. Current smokers also more or less lived in poor neighbourhoods with nonproblematic neighbourhood type and they were non-drinkers of alcohol in past month. All of the factors mentioned above associated significantly with cigarette smoking between 2002 and 2005.

With respect to dagga/other drugs between 2002 and 2005, the same pattern observed in cigarette smoking was noticed regarding age, sex of the respondents, race and educational level. In addition, those who grew up in urban areas and in predominantly Coloured neighbourhoods, out of school, attending problematic schools in 2005, non-participant in prosocial activities, those living in poor neighbourhoods, who grew up in non-problematic neighbourhoods, and current drinkers of alcohol at the baseline were more of current dagga/others drugs in the study area. All of the factors mentioned above were statistically significantly related to dagga/other drugs' smoking behaviour between 2002 and 2005.

Table 5: Binary logistic regression results showing the determinants of smoking behaviour-cigarette and dagga/other drugs (CAPS 2002-2005)

\begin{tabular}{|c|c|c|c|c|}
\hline \multirow[t]{3}{*}{ Variable(s) } & Wave 1 (2002) & Wave 3 (2005) & Wave 1 (2002) & Wave 3 (2005) \\
\hline & \multicolumn{2}{|c|}{ Cigarette Smoking } & \multicolumn{2}{|c|}{ Smoking of Dagga/other drugs } \\
\hline & Odds Ratio & Odds Ratio & Odds Ratio & Odds Ratio \\
\hline \multicolumn{5}{|c|}{ Independent variables } \\
\hline \multicolumn{5}{|l|}{ Age } \\
\hline Less than 18 years & $\mathrm{RC}$ & $\mathrm{RC}$ & $\mathrm{RC}$ & $\mathrm{RC}$ \\
\hline 18 years and above & $1.07(0.84-1.38)$ & $1.36(0.89-2.07)$ & $1.04(0.64-1.71)$ & $\begin{array}{ll}0.25 * * * & (0.17- \\
0.36) & \\
\end{array}$ \\
\hline \multicolumn{5}{|c|}{ Sex of the respondents } \\
\hline Male & $\mathrm{RC}$ & $\mathrm{RC}$ & $\mathrm{RC}$ & $\mathrm{RC}$ \\
\hline Female & $\begin{array}{ll}0.46^{* * * *} \quad(0.37- \\
0.56)\end{array}$ & $\begin{array}{ll}0.56 * * * & (0.41- \\
0.78) & \\
\end{array}$ & $\begin{array}{ll}0.27 * * * & (0.17- \\
0.44) & \\
\end{array}$ & - \\
\hline \multicolumn{5}{|c|}{ Race/ population group } \\
\hline Black/African & $\mathrm{RC}$ & $\mathrm{RC}$ & $\mathrm{RC}$ & $\mathrm{RC}$ \\
\hline Coloured & $\begin{array}{ll}5.98 * * * & (2.32- \\
15.40) & \\
\end{array}$ & $1.61(0.36-7.20)$ & $\begin{array}{ll}0.40 \quad(0.07- \\
2.24) & \\
\end{array}$ & $\begin{array}{l}1.19 \\
4.60)\end{array}$ \\
\hline White & - & $0.44(0.10-1.86)$ & - & $\begin{array}{ll}0.91 & (0.18- \\
4.53) & \end{array}$ \\
\hline Indian/Asian & $2.08(0.71-6.12)$ & $1.11(0.16-7.71)$ & $\begin{array}{ll}0.20 \quad(0.03- \\
1.24)\end{array}$ & $\begin{array}{ll}1.73 & (0.14- \\
21.13) & \\
\end{array}$ \\
\hline \multicolumn{5}{|l|}{ Educational level } \\
\hline Primary & $\mathrm{RC}$ & $\mathrm{RC}$ & $\mathrm{RC}$ & $\mathrm{RC}$ \\
\hline Secondary & $1.21(0.92-1.60)$ & $\begin{array}{ll}0.60 * * & (0.40- \\
0.91) & \\
\end{array}$ & - & - \\
\hline Tertiary & $\begin{array}{ll}0.53 * * & (0.30- \\
0.95) & \\
\end{array}$ & $\begin{array}{ll}0.60 * * & (0.40- \\
0.91) & \\
\end{array}$ & - & - \\
\hline \multicolumn{5}{|l|}{ Current work status } \\
\hline Yes & $\mathrm{RC}$ & $\mathrm{RC}$ & $\mathrm{RC}$ & $\mathrm{RC}$ \\
\hline No & - & $0.95(0.61-1.46)$ & - & - \\
\hline \multicolumn{5}{|l|}{ Contextual Factors } \\
\hline \multicolumn{5}{|l|}{ Religious affiliation } \\
\hline None & $\mathrm{RC}$ & $\mathrm{RC}$ & $\mathrm{RC}$ & $\mathrm{RC}$ \\
\hline Christianity & $\begin{array}{ll}0.70 * * \quad(0.49- \\
0.99)\end{array}$ & $0.71(0.37-1.35)$ & - & - \\
\hline Islam & $\begin{array}{l}1.58 * * \quad(1.02- \\
2.43)\end{array}$ & $1.32(0.58-3.03)$ & - & - \\
\hline Others & $0.71(0.48-1.05)$ & $0.93(0.48-1.82)$ & - & - \\
\hline \multicolumn{5}{|c|}{ Childhood place of residence } \\
\hline Urban & $\mathrm{RC}$ & $\mathrm{RC}$ & $\mathrm{RC}$ & $\mathrm{RC}$ \\
\hline Rural & $1.03(0.72-1.48)$ & $\begin{array}{ll}0.45^{* *} \quad(0.22- \\
0.90) & \\
\end{array}$ & $\begin{array}{ll}0.67 & (0.29- \\
1.54) & \\
\end{array}$ & $\begin{array}{ll}0.46 * * & (0.24- \\
0.88) & \\
\end{array}$ \\
\hline \multicolumn{5}{|c|}{$\begin{array}{ll}\begin{array}{l}\text { Neighbourhood } \\
\text { concentration }\end{array} & \text { racial } \\
\end{array}$} \\
\hline African & $\mathrm{RC}$ & $\mathrm{RC}$ & $\mathrm{RC}$ & $\mathrm{RC}$ \\
\hline
\end{tabular}




\begin{tabular}{|c|c|c|c|c|}
\hline Coloured & $0.81(0.32-2.08)$ & $\begin{array}{ll}2.97 & (0.64- \\
13.74) & \end{array}$ & $\begin{array}{ll}4.00 & (0.71- \\
22.52) & \end{array}$ & $\begin{array}{ll}1.15 & (0.30- \\
4.44) & \end{array}$ \\
\hline White & $0.92(0.33-2.56)$ & $\begin{array}{ll}4.33^{* *} & (1.03- \\
18.17) & \end{array}$ & $\begin{array}{ll}4.70 & (0.89- \\
24.71) & \end{array}$ & $0.89(0.20-4.02)$ \\
\hline \multicolumn{5}{|l|}{ School attendance } \\
\hline Yes & $\mathrm{RC}$ & $\mathrm{RC}$ & $\mathrm{RC}$ & $\mathrm{RC}$ \\
\hline No & $\begin{array}{ll}3.42 * * * & (2.68- \\
4.36) & \\
\end{array}$ & $1.25(0.61-2.57)$ & $\begin{array}{ll}2.22 * * * & (1.33- \\
3.72) & \\
\end{array}$ & $\begin{array}{ll}1.67 * * & (1.13- \\
2.48) & \\
\end{array}$ \\
\hline \multicolumn{5}{|c|}{ Participation in prosocial activities } \\
\hline Non-participants & $\mathrm{RC}$ & $\mathrm{RC}$ & $\mathrm{RC}$ & $\mathrm{RC}$ \\
\hline Participants & $0.83(0.68-1.03)$ & $0.90(0.64-1.28)$ & $\begin{array}{ll}0.43 * * * & (0.27- \\
0.67) & \\
\end{array}$ & $\begin{array}{ll}0.66 * * & (0.49- \\
0.91) & \\
\end{array}$ \\
\hline \multicolumn{5}{|l|}{ Neighbourhood SES } \\
\hline Affluent & $\mathrm{RC}$ & $\mathrm{RC}$ & $\mathrm{RC}$ & $\mathrm{RC}$ \\
\hline Poor & $0.88(0.70-1.11)$ & $0.94(0.64-1.39)$ & - & $\begin{array}{ll}0.79 & (0.56- \\
1.13) & \\
\end{array}$ \\
\hline \multicolumn{5}{|c|}{ Childhood neighbourhood type } \\
\hline Non-problematic & $\mathrm{RC}$ & $\mathrm{RC}$ & $\mathrm{RC}$ & $\mathrm{RC}$ \\
\hline Problematic & $\begin{array}{ll}1.51 * * * & (1.22- \\
1.88) & \\
\end{array}$ & $1.16(0.80-1.68)$ & $\begin{array}{ll}2.04 * * * & (1.34- \\
3.10) & \\
\end{array}$ & $\begin{array}{ll}1.65 * * * & (1.20- \\
2.26) & \\
\end{array}$ \\
\hline \multicolumn{5}{|c|}{ Consumed Alcohol in past month } \\
\hline Yes & $\mathrm{RC}$ & $\mathrm{RC}$ & $\mathrm{RC}$ & $\mathrm{RC}$ \\
\hline No & $\begin{array}{l}0.18^{* * * *} \quad(0.14- \\
0.22)\end{array}$ & $\begin{array}{l}5.67 * * * \quad(3.92- \\
8.18)\end{array}$ & $\begin{array}{l}0.29 * * * \quad(0.18- \\
0.45)\end{array}$ & $\begin{array}{l}3.14 * * * \quad(2.24- \\
4.41)\end{array}$ \\
\hline Constant & $\begin{array}{ll}0.46 * * * & (0.27- \\
0.78) & \\
\end{array}$ & $\begin{array}{l}0.11 * * * * \quad(0.04- \\
0.27)\end{array}$ & $\begin{array}{l}0.07 * * * \quad(0.03- \\
0.16)\end{array}$ & $\begin{array}{ll} & 0.03 * * * \\
0.07) & (0.02- \\
\end{array}$ \\
\hline Log likelihood & -1271.5172 & -481.52669 & -369.80496 & -617.97758 \\
\hline Number of observation & $\mathbf{3 , 1 3 4}$ & 1,129 & 3,168 & 3,173 \\
\hline Prob $>\mathrm{Chi}^{2}$ & 0.0000 & 0.0000 & 0.0000 & 0.0000 \\
\hline${\text { Pseudo } \mathbf{R}^{2}}^{2}$ & 0.2840 & 0.1950 & 0.1978 & $\mathbf{0 . 1 5 3 6}$ \\
\hline
\end{tabular}

Significant at $* * * \mathrm{p}<0.01 ; * * \mathrm{p}<0.05$; $\mathrm{RC}=$ Reference Category; Figures in parentheses are $95 \%$ confidence interval Dagga $=$ Marijuana

Table 5 presents the determinants of smoking cigarette and dagga/other drugs between 2002 and 2005. With respect to current cigarette smoking, females were significantly less likely to be current cigarette smokers (O.R: 0.46 and $0.56 ; \mathrm{p}<0.01)$ when compared to their male counterparts while Coloured respondents were significantly more likely to be current smokers at the baseline (O.R: 5.98; p<0.01) when compared to their African counterparts. Having secondary education significantly reduces the odds of being a current smoker at the third wave while having tertiary education qualification reduce the odds of currently smoking cigarette at both first and third wave of data collection $(p<0.05)$ when compared to those with primary level of education. With respect to the influence of contextual factors, Christians had lower odds of being current smokers of cigarette (O.R: 0.70; $\mathrm{p}<0.05)$ while those with Islamic religion had twice the odds of being current smokers when compared to those without any religious affiliation in 2002.

Those who grew up in a rural area had significantly lower odds (O.R: $0.45 ; \mathrm{p}<0.05)$ when compared to those who grew up in an urban setting. With respect to neighbourhood racial concentration, those in predominantly White neighbourhoods had higher odds of reporting current cigarette smoking (O.R: 4.33 ; $<<0.05)$ when compared to their African counterparts in 2005. Those who are out of school had significantly higher odds of being current cigarette smokers especially at the baseline when compared to those in school. Those who grew up in a problematic environment had significantly higher odds (O.R: $1.51 ; \mathrm{p}<0.01)$ at the baseline of being current smokers while those who were not current drinker of alcohol in 2002 had significantly lower odds of being current cigarette smokers although at the third wave, those who did not consume alcohol in past month had significantly higher odds of being current cigarette smokers (O.R: 5.67; p<0.01).

However, in the case of dagga/other drugs, those who were 18 years and above had lower odds of smoking dagga/other drugs in 2005 (O.R: 0.25; p<0.01) when compared to their younger counterparts at the baseline, females were significantly less likely to be current smokers when compared to their male counterparts (O.R: 0.27; p<0.01). Also, those in grew up in rural areas were 54\% less likely to be current smokers of dagga/other drugs (p<0.05). Moreover, being out of school increased the odds of being current smokers of dagga/other drugs between 2002 and 2005 (O.R: 2.22 ; $\mathrm{p}<0.01$; O.R: $1.67 ; \mathrm{p}<0.05)$. Participating in prosocial activities significantly reduced the odds of being current smokers 
of dagga/other drugs when compared to non-participants between 2002 and 2005. Those who grew up in problematic neighbourhoods had significantly higher odds of smoking dagga/other drugs $(\mathrm{p}<0.01)$. Lastly, those who did not consume alcohol in past month at the baseline were significantly less likely to be current smokers of dagga/other drugs when compared to alcohol users $(\mathrm{p}<0.01)$ while at the third wave of data collection, those who did not consume alcohol in past month were significantly more likely to be current smokers of dagga/other drugs in the study area when compared to alcohol consumers (O.R: 3.14; p<0.01) in 2005.

It is clear from Table 6 that across the three waves, those who were not current smokers of cigarette had a decreased odds of reporting smoking-related illnesses in the study area and the relationship is statistically significant $(\mathrm{p}<0.05)$. Specifically, in Wave 1, adolescents and young South Africans living in the Cape Peninsula, who were not current smokers of cigarette in 2002 were $47 \%$ less likely to report smoking-related illnesses $(\mathrm{p}<0.05)$. At the second Wave in 2002/2003, adolescents and young South Africans living in the study area, who were not current smokers of cigarette, had $57 \%$ lesser odds of reporting smoking-related illnesses $(\mathrm{p}<0.05)$; while at the third Wave, non-smokers of cigarette in past month were significantly less likely to report smoking-related illnesses $(\mathrm{p}<0.05)$.

Table 6: Binary logistic regression predicting the odds of experiencing smoking-related illnesses (CAPS 2002-2005)

\begin{tabular}{|l|l|l|l|}
\hline Correlate & Wave 1 & Wave 2 & Wave 3 \\
\hline $\begin{array}{l}\text { Smoked cigarette in past } \\
\text { month? }\end{array}$ & Odds Ratio & Odds Ratio & Odds Ratio \\
\hline Yes & RC & RC & RC \\
\hline No & $0.53^{* *}(0.30-0.92)$ & $0.43^{* *}(0.20-0.94)$ & $0.46^{* *}(0.24-0.86)$ \\
\hline Log likelihood & -183.94974 & -84.905343 & -142.1233 \\
\hline No. of Observation & 285 & 128 & 213 \\
\hline Prob $>$ chi $^{2}$ & 0.0252 & 0.0340 & 0.0151 \\
\hline Pseudo $\mathrm{R}^{2}$ & 0.0134 & 0.0258 & 0.0203 \\
\hline
\end{tabular}

Significant at $* * * \mathrm{p}<0.01 ; \mathrm{p}<0.05$

The figures in parentheses are confidence interval; $\mathrm{RC}=$ Reference Category

\section{DISCUSSION}

From the results of the analyses in this paper, some points are worthy of emphasis. Although many of the past studies were cross-sectional in design, the findings in this paper, using a panel data, confirm some of the results in earlier studies. In spite of strong and powerful legislation in relation to smoking, adolescents below the statutory age of 18 years are smoking cigarette and dagga/other drugs in the study area. The prevalence rate of past month cigarette smokers $(25 \%$ and $36 \%$ ) in this study is a little more than what had been previously reported in South Africa, the previous prevalence rate ranging from $11 \%$ to $21 \%[24,15,16,26]$.

There is an indication that uptake of smoking behaviour increases with age as the proportion of the young respondents who were current smokers of cigarette at the baseline increased from one-quarter to more than one-third at the third wave of data in 2005, and those who were current smokers of dagga (Marijuana) or other drugs increased from $3 \%$ to $6 \%$ between 2002 and 2005. This is in line with past studies in another setting [31]. The findings in this study that more males smoke cigarette than their female counterparts and that smoking prevalence is higher in urban areas in comparison to the rural areas, agreed with what had been found in earlier studies [24, 26].

Adolescent of Coloured racial origin were found to have the highest proportion of current smokers contrary to what was found by King and colleagues (2003) where he asserted that White students had the highest proportion of cigarette smokers when compared to other racial groups [34]. Caution ought to be exercised however with regard to this finding because CAPS took place in predominantly Coloured-dominated neighbourhoods and that may have accounted for the high prevalence recorded among the Coloureds. When predominant racial groupings at the residential level was factored into the equation, those adolescents and young adults living in predominantly White neighbourhoods had higher odds of reporting current cigarette smoking in comparison to their African counterparts in 2005. The Whites had been found to be the most-inclined to be smokers [25]. There is an indication that the higher the level of education, the lower of the odds of being current cigarette smokers. Current smokers also more or less lived in poor neighbourhoods thereby confirming and echoing the fact that there is an association between poverty and high level of smoking [20]. Also, van Walbeek (2002) had earlier found that poor people spend a larger proportion of their income on cigarette [4].

Those who are out of school had significantly higher odds of being current cigarette and Marijuana smokers especially at the baseline when compared to those in school. This makes a lot of sense in the context of a lot of spare time to experiment with different things including smoking and because of the unrestricted exposure to different types of 
friends who are sometimes gatekeepers of deviant behaviours in their community. It was found that those who grew up in a problematic environment had significantly higher odds $(\mathrm{O} . \mathrm{R}: 1.51 ; \mathrm{p}<0.01)$ at the baseline of being current smokers. This relates to the presence of adults (parents inclusive) who are positive role models in the immediate environment. This lends credence to the fact that although peer influence is an important factor to reckon, influence of positive role models in terms of non-smokers and health-promoting adults is more crucial to the fight against high prevalence of smoking [34, $28,26,33]$ among young people in the study area.

There is a kind of mixed result between alcohol consumption and smoking in the study although there seems to be some kind of clustering between the two behaviours as found in different settings [16, 31]. Those who were not current drinker of alcohol in 2002 had significantly lower odds of being current cigarette smokers at the baseline, but at the third wave, those who did not consume alcohol in past month had significantly higher odds of being current cigarette smokers (O.R: 5.67; p<0.01). Also, participating in prosocial activities significantly reduced the odds of being current smokers of dagga/other drugs when compared to non-participants between 2002 and 2005. Therefore, using the avenue of prosocial groupings to disseminate useful and health-enhancing information should be of high priorities in the study area.

In line with past studies linking smoking to ill health and premature death $[6,5,10]$, this study found that those who were not current smokers of cigarette at the baseline had a decreased odds of reporting smoking-related illnesses (tuberculosis and other respiratory illnesses) in the study area at a later wave of data collection and the relationship is statistically significant $(\mathrm{p}<0.05)$ in all the three wave of data collection. This could be an indication of an immediate manifestation and consequence of their smoking behaviours, and promoting health-enhancing behaviours among the adolescents and the young people studied would be beneficial on the short and long run. Despite the fact that CAPS dataset was not designed primarily for this study and that this paper rely on self-reported responses to some retrospective questions which are subject to biases and collected between 2002 and 2005, the longitudinal nature of the data provides a unique opportunity to establish causal relationships between the independent and outcome variables of interest which hitherto is not possible through cross-sectional study design.

\section{CONCLUSION}

The study concludes that anti-smoking campaign and legislation is failing among adolescents and young people living in the study area as young people below the statutory adult age of 18 years are indulging in smoking of cigarette and dagga/other drugs, and that both personal (such as age, sex, and education) and neighbourhood/environmental (religious affiliation, childhood place of residence, neighbourhood residential arrangement, school attendance, participation in prosocial activities, child neighbourhood type and consumption of alcohol) factors predispose adolescents in Cape Town to cigarette and dagga/other drugs' smoking behaviour. Lastly, smoking of cigarette has both immediate and long-term negative health consequences for the young people as indicated in this study.

\subsection{Recommendations}

Recommendations that will reduce the uptake of smoking habit among young people in the study area include proper monitoring of legislation regarding smoking and other related matters. Although South Africa has been referred to as a global leader in developing appropriate legislation for tobacco control, there seems to be a loophole in the area of implementation and monitoring. To protect the health of young people in the Cape Peninsula in particular and South Africa in general, there is need on the part of the government to put in place appropriate authorities that will monitor the enforcement of the various legislations designed to protect the health and invariably the future of young South Africans. Effective intervention programmes that will discourage smoking behaviour among adolescents and young adults in the study area would be beneficial to the health of the South Africans on the long run, especially considering the high incidence on non-communicable diseases in the country and the fact that the trend may continue into the future if there is no appropriate interventions that would target the significant personal and contextual factors. Moreover, creation of a society with positive role models would be an advantage towards stemming the increasing prevalence of cigarette smoking among young South Africans. There should be appropriate information, education and communication (IEC) programmes that would raise awareness of the negative consequences of smoking in the study area.

\section{ACKNOWLEDGMENT}

The Cape Area Panel Study Waves 1-2-3 were collected between 2002 and 2005 by the University of Cape Town and the University of Michigan, with funding provided by the US National Institute for Child Health and Human Development and the Andrew W. Mellon Foundation. Wave 4 was collected in 2006 by the University of Cape Town, University of Michigan and Princeton University. Major funding for Wave 4 was provided by the National Institute on Aging through a grant to Princeton University, in addition to funding provided by NICHD through the University of Michigan. 


\section{REFERENCES}

[1] WHO (2002): "Smoking Statistics 2002" available http://www.wpro.who.int/media_centre/fact_sheets/fs_20020528.htm accessed on March 23, 2014.

[2] Mathers, C.D. and Loncar, D. (2006): "Projections of global mortality and burden of disease from 2002 to 2030". PLoS Medicine, 2006, 3:e442.

[3] Mackay, J.E. (2002): "The Tobacco Atlas". Geneva: World Health Organization 2002 available at http://www.who.int/tobacco/en/atlas 11.pdf accessed on March 23, 2014.

[4] van Walbeek, C. (2002): "Recent Trends in Smoking Prevalence in South Africa- Some Evidence from AMPS Data". S Afr Med J 2002; Vol. 92, No. 6, pp. 468-472.

[5] WHO (2003): "Social determinants of health: the solid facts". 2nd Edition in Wilkinson, R. and Marmot, M. (eds.), World Health Organization 2003.

[6] Yach, D. and Townshend, G.S. (1988): "Smoking and health in South Africa". South African Medical Journal 1988, 73(7): 391-399.

[7] Ezzati, M. and Lopez, A. (2004): "Smoking and oral tobacco use" In: Ezzati, M., Lopez, A.D., Rodgers, A. and Murray, C.J.L (Eds.). Comparative quantification of health risks: global and regional burden of disease attributable to selected major risk factors", Volume 1, Chapter 11. Geneva: World Health Organization, 2004: 497-596.

[8] Bjartveit, K. and Tverdal, A. (2005): "Health Consequences of Smoking 1-4 cigarettes per day", Tobacco Control. 2005; 14:315-20.

[9] Line, H. et al. (2007): "Tobacco smoke, indoor air pollution and tuberculosis: a systematic review and metaanalysis". PLoS Medicine 2007, 4:e20.

[10] OECD (2013): "Health at a Glance 2013: OECD Indicators", OECD Publishing accessible at http://dx.doi.org/10.1787/health_glance-2013-en.

[11] WHO (2010): "Global estimate of the burden of disease from second-hand smoke". Geneva: World Health Organization, 2010.

[12] WHO (2009): "Global health risks: mortality and burden of disease attributable to selected major risks". Geneva: World Health Organization, 2009.

[13] Mattias, O. et al. (2011): "Worldwide burden of disease from exposure to second-hand smoke: a retrospective analysis of data from 192 countries". The Lancet, 2011, 377:139-146.

[14] Panday, S., Reddy, S.P., Ruiter, R.A.C., Bergstrom, E. and De Vries, H. (2007): "Determinants of smoking among adolescents in the Southern Cape- Karoo region, South Africa," Health Promotion International, Vol. 22, No. 3, pp. 207-217, June 2007 doi:10.1093/heapro/dam018.

[15] Swart, D., Reddy, P., Panday, S., Philip, J., Naidoo, N. and Ngobeni, N. (2004): “The 2002 Global Youth Tobacco Survey (GYTS): The 2nd GYTS in South Africa (SA)- A Comparison between GYTS (SA) 1999 and GYTS (SA) 2002", Medical Research Council, Cape Town.

[16] O'Hara, O.R. (2008): “The association of adolescent smoking with stress and coping in Pretoria High School: A Qualitative Study". A research report submitted to the Faculty of Health Sciences, University of the Witwatersrand, Johannesburg in partial fulfillment of the requirements for the degree of Master of Public Health (Health Policy and Management), 2008.

[17] Statistics South Africa (2004): "Provincial Profile 1999: Western Cape" Statistics South Africa 2004. Pretoria: Statistics South Africa, 2004. 92p. [Report No. 00-91-01 (1999)]

[18] Bradshaw, D., Groenewald, P., Laubscher, R., Nannan, N., Nojilana, B., Norman, P., Pietersen, D. and Schneider, M. (2003): “Initial Burden of Disease Estimates for South Africa, 2000". South African Medical Research Council, Cape Town.

[19] Healthy Active Kids South Africa (2010): "Report Card 2010: Report Card on the physical activity, nutrition and tobacco use for South African children and youth" available at http://www.globalpa.org.uk/downloads/healthy-active-kids-report-2010.pdf and accessed on 23/03/2014.

[20] Panday, S., Reddy, S.P. and Bergstrom, E. (2003): "A qualitative study on the determinant of smoking behavior among adolescents in South Africa". Scand J Public Health, May 2003, Vol. 31, No. 3: 204-210.

[21] Panday, S., Reddy, S.P., Ruiter, R.A.C., Bergstrom, E. and De Vries, H. (2005): "Determinants of smoking cessation among adolescents in South Africa”, Health Education Research, Theory and Practice Vol. 20, No. 5, pp. 586-599.

[22] Peltzer, K. (2001): "Tobacco Smoking in Black and White South Africans". East Afr Med J 2001; 78:115-18.

[23] Brook, J.S., Morojele, N.K., Brook, D.W., Zhang, C. and Whiteman, M. (2006): "Personal, interpersonal and Cultural predictors of stages of Cigarette smoking among adolescents in Johannesburg, South Africa", Tobacco Control 2006; 15: i48-i53; doi: 10.1136/tc.2005.014878

[24] Madu and Matla (2004): "Illicit drug use, cigarette smoking and alcohol drinking behavior among a sample of high school adolescents in the Pietersburg area of the Northern Province, South Africa", Journal of 
Community Agency for Social Enquiry (2002): "Youth 2002: a study of youth in South Africa". A Report for the Royal Netherlands Embassy. Johannesburg: CASE; 2002 accessible at http://www.case.org.za.

[26] Reddy, S.P., James, S., SewPaul, R., Koopman, F., Funani, N.I., Sifunda, S., Josie, J., Masuka, P., Kambaran, N.S., Omardien, R.G. Umthente Uhlaba Usamila (2008): "The South African Youth Risk Behaviour Survey 2008". Cape Town: South African Medical Research Council, 2010.

[27] WHO (2004): “Global Status report on alcohol 2004”. Geneva: World Health Organization.

[28] Swart, D., Panday, S., Reddy, S.P., Bergstrom, E. and de Vries, H. (2006): "Access point analysis: what do adolescents say about tobacco control programmes?", Health Education Research 2006, 21(3), 393-406.

[29] van Heerden, M.S., Grimsrud, A.T., Seedat, S., Myer, L., Williams, D.R. and Stein, D.J. (2009): "Patterns of substance use in South Africa: results from the South African Stress and Health Study". South African Medical Journal 2009, 99 (5 Pt 2), 358-366.

[30] Schmidt, L. (2013): "The path to tobacco addiction starts at very young ages", accessed from http://www.tobaccofreekids.org/research/factsheets/pdf/0127.pdf on 23/03/2014.

31.

[31] Faeh, D., Viswanathan, B., Chiolero, A., Warren, W. and Bovet, P. (2006): "Clustering of smoking, alcohol drinking and cannabis use in adolescents in a rapidly developing country", BMC Public Health 2006, June 27; 6:169.

[32] Guindon, G. (2009): “The impact of tobacco prices on smoking onset in Vietnam: duration analysis of retrospective data", Centre for Health Economics and Policy Analysis, McMaster University, Hamilton, Ontario, Canada available at https://editorialexpress.com/cgibin/conference/download.cgi?db_name=CEA2010\&paper_id=838 and accessed on 02/04/2014.

[33] Pahl, K., Brook, D.W., Morojele, N.K. and Brook, J.S. (2010): "Nicotine dependence and problem behaviours among urban South African adolescents". Journal of Behavioural Medicine 2010, 33(2), 101-109.

[34] King, G., Flisher, A.J., Mallett, R., Graham, J., Lombard, C., Rawson, T., Morojele, N.K. and Muller, M. (2003): "Smoking in Cape Town: Community Influences on Adolescent Tobacco Use". Preventive Medicine, Vol. 36, Issue 1, January 2003, Pages 114-123.

[35] Lam David, Cally Ardington, Nicola Branson, Anne Case, Murray Leibbrandt, Alicia Menendez, Jeremy Seekings and Meredith Sparks (2008): "The Cape Area Panel Study: A Very Short Introduction to the Integrated Waves 1-2-3-4 Data". The University of Cape Town, October 2008.

[36] Dinkelman, T., Lam, D. and Leibbrandt, M. (2007): "Household and community income, economic shocks and risky sexual behaviour of young adults: evidence from the Cape Area Panel Study 2002 and 2005”, AIDS. 2007 November; 21 (Supplement 7): S49-S56.

[37] Alderman, H., Behrman, J.R., Kohler, H-P., Maluccio, J.A. \& Watkin, S.C. (2001): "Attrition in Longitudinal Household Survey Data", Demographic Research, Volume 5, Article 4, Pp. 79-124.

[38] StataCorp. (2011): "Stata: Release 12", Statistical Software. College Station, TX: StataCorp LP.

[39] Lam David, Cally Ardington, Nicola Branson, Anne Case, Murray Leibbrandt, Alicia Menendez, Jeremy Seekings and Meredith Sparks (2008): "Overview and Technical Documentation Waves 1-2-3-4 (2002-2006)". The University of Cape Town, October 2008.

[40] Adato, M. \& Haddad, L. (2002): "Targeting Poverty through Community-Based Public Works Programmes: Experience from South Africa”, Journal of Development Studies, 38: 3, pp. 1-36. 


\section{Appendix \\ Operational Definitions of variables}

\begin{tabular}{|c|c|c|}
\hline Variable(s) & Items & Measurement in this study \\
\hline \multicolumn{3}{|l|}{ Dependent variables } \\
\hline Smoked cigarette in past month & $\begin{array}{l}\text { Consumed cigarette in the past } \\
\text { month? }\end{array}$ & $\mathrm{No}=0 ; \mathrm{Yes}=1$ \\
\hline $\begin{array}{l}\text { Smoked dagga/other drugs in past } \\
\text { month }\end{array}$ & $\begin{array}{l}\text { Consumed cigarette in the past } \\
\text { month? }\end{array}$ & $\mathrm{No}=0 ; \mathrm{Yes}=1$ \\
\hline $\begin{array}{l}\text { Experience of smoking-related } \\
\text { illnesses (self-reported illnesses) }\end{array}$ & $\begin{array}{l}\text { What are your health } \\
\text { problems/disabilities? }\end{array}$ & $\begin{array}{l}\text { Those who reported tuberculosis and } \\
\text { other respiratory illnesses }=1 \text {; Those } \\
\text { without these illnesses }=0\end{array}$ \\
\hline \multicolumn{3}{|c|}{ Socio-economic/demographic and personal factors } \\
\hline Age & Age at last birthday & $\begin{array}{l}\text { Given in single years } 14,15,16 \ldots 25 \\
\text { years (continuous variable) but } \\
\text { categorized into }<18 \text { years }=1 ; \geq 18 \\
\text { years }=2\end{array}$ \\
\hline Sex & Sex & Male $=1 ;$ Female $=2$ (Dichotomous $)$ \\
\hline Race/ population group & $\begin{array}{l}\text { The racial/population group } \\
\text { according to traditional apartheid } \\
\text { categories }\end{array}$ & $\begin{array}{l}\text { Categorical (Black/African }=1 ; \\
\text { Coloured }=2 ; \text { White }=4 . \text { (N.B: } \\
\text { Indians/Asians }(3) \text { are very few in } \\
\text { number (less than } 1 \% \text { ) and are } \\
\text { sometimes excluded by the system } \\
\text { during the multivariate analysis). }\end{array}$ \\
\hline Educational level & Highest educational level attained & $\begin{array}{l}\text { Categorical from no formal } \\
\text { education to others (Recoded to } \\
\text { Primary }=1 ; \text { Secondary }=2 \text { and } \\
\text { Tertiary }=3 \text { ). }\end{array}$ \\
\hline Work status & Do you currently have a job? & Dichotomous $($ Yes $=1 ; \mathrm{No}=2)$ \\
\hline \multicolumn{3}{|c|}{ Contextual factors: facilitators or impediments } \\
\hline Religious affiliation & Faith group they belong to & $\begin{array}{l}\text { Categorical (Christian }=1 ; \text { Catholic } \\
=2 ; \text { Muslim }=3 ; \text { None }=4 \text { and Other } \\
=5)\end{array}$ \\
\hline $\begin{array}{l}\text { Type of place spent most of life } \\
\text { (childhood place of residence) }\end{array}$ & $\begin{array}{l}\text { Type of place where the young } \\
\text { people spent most of their lives up to } \\
\text { age } 14 \text { years }\end{array}$ & $\begin{array}{l}\text { Categorical (Formal urban }=1 ; \\
\text { Informal urban }=2 ; \text { Commercial } \\
\text { farm }=3 ; \text { Rural under chief }=4 ; \\
\text { Other rural }=5 ; \text { and Others }=6 \text { ) } \\
\text { Recoded to Urban }=1 \text { and Rural }=2\end{array}$ \\
\hline Neighbourhood racial concentration & $\begin{array}{l}\text { Most numerous population group in } \\
\text { the sub-place }\end{array}$ & $\begin{array}{l}\text { Categorical }(\text { Africans }=1 ; \text { Coloureds } \\
=2 \text { and Whites }=3)\end{array}$ \\
\hline Family structure (Composite) & $\begin{array}{l}\text { Are your parents (mother; father) } \\
\text { living with you in this household? }\end{array}$ & $\begin{array}{l}\text { Generated No parent }=1 ; \text { Single } \\
\text { parent }=2 \text { and Both parents }=3\end{array}$ \\
\hline $\begin{array}{l}\text { School attendance (Current school } \\
\text { enrolment) }\end{array}$ & Are you currently in school? & Dichotomous (Yes $=1 ;$ No $=2$ ) \\
\hline School environment (Composite) & $\begin{array}{l}\text { How do you describe the type of } \\
\text { school you are attending? } 13 \\
\text { different types of problem in the } \\
\text { school environment were } \\
\text { highlighted. }\end{array}$ & $\begin{array}{l}\text { Problematic school environment }=1 \\
\text { and Non-problematic school } \\
\text { environment }=2 \text {. }\end{array}$ \\
\hline $\begin{array}{l}\text { Participation in pro-social activities } \\
\text { (Composite) }\end{array}$ & $\begin{array}{l}\text { Belong to? } \\
\text { (i) sports group or team? } \\
\text { (ii) a study group? } \\
\text { (iii) a religious group? } \\
\text { (iv) a music group? } \\
\text { (v) } \quad \text { some other youth group? }\end{array}$ & $\begin{array}{l}\text { Generated from responses to five } \\
\text { questions }(\text { Yes }=1 ; \text { No }=2) \text {. } \\
\text { Recoded to Participants in at least } \\
\text { one }=1 \text { and Non-participants in any = } \\
2 .\end{array}$ \\
\hline
\end{tabular}




\begin{tabular}{|l|l|l|}
\hline Neighbourhood SES/poverty & $\begin{array}{l}\text { Using 1996 South African census } \\
\text { classification. The magisterial } \\
\text { districts were categorised into } \\
\text { affluent or poor based on the } 1995 \\
\text { October Household Survey (see } \\
\text { Adato and Haddad 2002 for details). }\end{array}$ & $\begin{array}{l}\text { Categorisation into 10 districts } \\
\text { namely: Bellville = 101; Goodwood } \\
\text { =102; Cape = 103; Simon's Town }= \\
104 ; \text { Wynberg }=105 ; \text { Mitchell's } \\
\text { Plain }=106 ; \text { Kuils River }=107 ; \\
\text { Somerset West }=110 ; \text { Strand }=111 ; \\
\text { and Malmesbury }=132 . \text { Recoded to } \\
\text { Affluent neighbourhood }=1 \text { and } \\
\text { Poor neighbourhood }=2\end{array}$ \\
\hline Childhood neighbourhood type & $\begin{array}{l}\text { Growing up: Was there a problem } \\
\text { drinker? Did someone use street } \\
\text { drugs? Did someone spend time in } \\
\text { jail? }\end{array}$ & $\begin{array}{l}\text { Categorical (Yes }=1 ; \text { No }=2) \\
\text { Recoded into problematic=1 and } \\
\text { non-problematic=2). }\end{array}$ \\
\hline Consumed alcohol in past month & Consumed alcohol in past month? & No=0; Yes=1 \\
\hline
\end{tabular}

Note: It has been found that in most developing countries, unemployment and poverty goes hand in hand [40]. The variable 'neighbourhood SES/poverty' was derived from the list of magisterial districts from which CAPS data was collected using census tract-level variables from the 1996 Census and 1995 October Household Survey. Affluent neighbourhoods coincided with areas with low unemployment (employment rate of between 5.8\%-9.8\%) and a higher percentage of people with at least Standard 10 (completed High School) education) while poor neighbourhoods coincided with areas having higher unemployment rates (employment rate of between 10.6\%-23.5\%) and a lower percentage of people with at least Standard 10 education. According to this classification, the following neighbourhoods were deemed to be affluent: Cape, Strand, Somerset West, Bellville, Wynberg and Simon's Town; while the poor neighbourhoods are Malmesbury, Kuils River, Goodwood and Mitchell's Plain. 